Article

\title{
On Uniquely 3-Colorable Plane Graphs without Adjacent Faces of Prescribed Degrees
}

\author{
Zepeng $\mathrm{Li}^{1, *}$, Naoki Matsumoto ${ }^{2}$, Enqiang $\mathrm{Zhu}{ }^{3}$, Jin $\mathrm{Xu}^{4}$ and Tommy Jensen ${ }^{5}$ \\ 1 School of Information Science and Engineering, Lanzhou University, Lanzhou 730000, China \\ 2 Research Institute for Digital Media and Content, Keio University, Tokyo 108-8345, Japan \\ 3 Institute of Computing Science and Technology, Guangzhou University, Guangzhou 510006, China \\ 4 School of Electronics Engineering and Computer Science, Peking University, Beijing 100871, China \\ 5 Department of Mathematics, Kyungpook National University, Daegu 41566, Korea \\ * Correspondence: lizp@lzu.edu.cn
}

Received: 30 July 2019; Accepted: 26 August 2019; Published: 1 September 2019

\begin{abstract}
A graph $G$ is uniquely $k$-colorable if the chromatic number of $G$ is $k$ and $G$ has only one $k$-coloring up to the permutation of the colors. For a plane graph $G$, two faces $f_{1}$ and $f_{2}$ of $G$ are adjacent $(i, j)$-faces if $d\left(f_{1}\right)=i, d\left(f_{2}\right)=j$, and $f_{1}$ and $f_{2}$ have a common edge, where $d(f)$ is the degree of a face $f$. In this paper, we prove that every uniquely three-colorable plane graph has adjacent $(3, k)$-faces, where $k \leq 5$. The bound of five for $k$ is the best possible. Furthermore, we prove that there exists a class of uniquely three-colorable plane graphs having neither adjacent $(3, i)$-faces nor adjacent $(3, j)$-faces, where $i, j$ are fixed in $\{3,4,5\}$ and $i \neq j$. One of our constructions implies that there exists an infinite family of edge-critical uniquely three-colorable plane graphs with $n$ vertices and $\frac{7}{3} n-\frac{14}{3}$ edges, where $n(\geq 11)$ is odd and $n \equiv 2(\bmod 3)$.
\end{abstract}

Keywords: plane graph; unique coloring; uniquely three-colorable plane graph; construction; adjacent $(i, j)$-faces

\section{Introduction}

Graph coloring is one of the most studied problems in graph theory, because it has many important applications [1-3]. The main aim of the problem is to assign colors to the elements of a graph, such as vertices, subject to certain constraints.

For a plane graph $G, V(G), E(G)$, and $F(G)$ are the sets of vertices, edges, and faces of $G$, respectively. The degree of a vertex $v \in V(G)$, denoted by $d_{G}(v)$, is the number of neighbors of $v$ in $G$. The degree of a face $f \in F(G)$, denoted by $d_{G}(f)$, is the number of edges in its boundary, cut edges being counted twice. When no confusion can arise, $d_{G}(v)$ and $d_{G}(f)$ are simplified as $d(v)$ and $d(f)$, respectively. A face $f$ is a $k$-face if $d(f)=k$ and a $k^{+}$-face if $d(f) \geq k$. Two faces $f_{1}$ and $f_{2}$ of $G$ are adjacent $(i, j)$-faces if $d\left(f_{1}\right)=i, d\left(f_{2}\right)=j$, and $f_{1}$ and $f_{2}$ have at least one common edge. Two distinct paths of $G$ are internally disjoint if they have no internal vertices in common. For other terminologies and notations in graph theory, we refer to [4].

A $k$-coloring of a graph $G$ is an assignment of $k$ colors to the vertices of $G$ such that no two adjacent vertices are assigned the same color. A graph $G$ is $k$-colorable if $G$ admits a $k$-coloring. The chromatic number of $G$, denoted by $\chi(G)$, is the minimum number $k$ such that $G$ is $k$-colorable. A graph $G$ is uniquely $k$-colorable if $\chi(G)=k$ and $G$ has only one $k$-coloring up to the permutation of the colors, where the coloring is called a unique $k$-coloring of $G$. In other words, all $k$-colorings of $G$ induce the same partition of $V(G)$ into $k$ independent sets, in which an independent set is called a color class of $G$. In addition, uniquely colorable graphs may be defined in terms of their chromatic polynomials, which was initiated by Birkhoff [5] for planar graphs in 1912 and for general graphs by Whitney [6] in 1932. 
A graph $G$ is uniquely $k$-colorable if and only if its chromatic polynomial is $k$ !. For a discussion of chromatic polynomials, see Read [7].

Uniquely colorable graphs were first studied by Harary and Cartwright [8] in 1968. They proved the following theorem.

Theorem 1 (Harary and Cartwright [8]). Let $G$ be a uniquely k-colorable graph. Then, for any unique $k$-coloring of $G$, the subgraph induced by the union of any two color classes is connected.

As a corollary of Theorem 1, it can be seen that a uniquely $k$-colorable graph $G$ has at least $(k-1)|V(G)|-\left(\begin{array}{c}k \\ 2\end{array}\right)$ edges. There are many references on uniquely colorable graphs [9-13].

Dailey [14] proved that the problem of determining whether a graph $G$ is uniquely colorable is NP-complete. However, it is still open for the case of planar graphs. Therefore, it is important to characterize the structure of uniquely colorable planar graphs.

Chartrand and Geller [10] in 1969 started to study uniquely colorable planar graphs. They proved that uniquely three-colorable planar graphs with at least four vertices contain at least two triangles, uniquely four-colorable planar graphs are maximal planar graphs, and uniquely five-colorable planar graphs do not exist. Aksionov [15] in 1977 improved the lower bound for the number of triangles in a uniquely three-colorable planar graph. He proved that a uniquely three-colorable planar graph with at least five vertices contains at least three triangles and gave a complete description of uniquely three-colorable planar graphs containing exactly three triangles. Li et al. [12] proved that if a uniquely three-colorable planar graph $G$ has at most four triangles, then $G$ has two adjacent triangles. Moreover, for any $k \geq 5$, they constructed a uniquely three-colorable planar graph with $k$ triangles and without adjacent triangles.

Let $G$ be a uniquely $k$-colorable graph. $G$ is edge-critical if $G-e$ is not uniquely $k$-colorable for any edge $e \in E(G)$. Obviously, if a uniquely $k$-colorable graph $G$ has exactly $(k-1)|V(G)|-\left(\begin{array}{c}k \\ 2\end{array}\right)$ edges, then $G$ is edge-critical. Mel'nikov and Steinberg [16] in 1977 asked to find an exact upper bound for the number of edges in an edge-critical uniquely three-colorable planar graph with $n$ vertices. In 2013, Matsumoto [17] proved that an edge-critical uniquely three-colorable planar graph has at most $\frac{8}{3} n-\frac{17}{3}$ edges and constructed an infinite family of edge-critical uniquely three-colorable planar graphs with $n$ vertices and $\frac{9}{4} n-6$ edges, where $n \equiv 0(\bmod 4)$. This upper bound was improved by Li et al. [13] to $\frac{5}{2} n-6$ when $n \geq 6$.

In this paper, we mainly prove Theorem 2.

Theorem 2. If $G$ is a uniquely three-colorable plane graph, then $G$ has adjacent $(3, k)$-faces, where $k \leq 5$. The bound five for $k$ is the best possible.

Furthermore, by using constructions, we prove that there exist uniquely three-colorable plane graphs having neither adjacent $(3, i)$-faces nor adjacent $(3, j)$-faces, where $i, j$ are fixed in $\{3,4,5\}$ and $i \neq j$. One of our constructions implies that there exists an infinite family of edge-critical uniquely three-colorable plane graphs with $n$ vertices and $\frac{7}{3} n-\frac{14}{3}$ edges, where $n(\geq 11)$ is odd and $n \equiv 2$ (mod 3). Our results further characterize the structure of the uniquely three-colorable plane graphs. The results can be used in optimal territorial distribution of mobile operators' transmitters.

\section{Proof of Theorem 2}

Now, we prove Theorem 2. First we give a useful Lemma 1.

Lemma 1. Let $G$ be a plane graph with three faces. If $G$ has no adjacent $(3, k)$-faces, where $k \leq 5$, then $|E(G)| \geq 2|F(G)|$.

Proof. We prove this by using a simple charging scheme. Since $G$ has no adjacent $(3, k)$-faces when $k \leq 5$, for any edge $e$ incident to a three-face $f, e$ is incident to a face of degree at least six. Let 
$\operatorname{ch}(f)=d(f)$ for any face $f \in F(G)$, and we call $\operatorname{ch}(f)$ the initial charge of the face $f$. Let initial charges in $G$ be redistributed according to the following rule.

Rule: For each three-face $f$ of $G$ and each edge $e$ incident with $f$, the $6^{+}$-face incident with $e$ sends $\frac{1}{3}$ charge to $f$ through $e$.

Denote by $c h^{\prime}(f)$ the charge of a face $f \in F(G)$ after applying the redistributed rule. Then:

$$
\sum_{f \in F(G)} c h^{\prime}(f)=\sum_{f \in F(G)} c h(f)=\sum_{f \in F(G)} d(f)=2|E(G)|
$$

On the other hand, for any three-face $f$ of $G$, since the degree of each face adjacent to $f$ is at least six, then by the redistributed rule, $c h^{\prime}(f)=\operatorname{ch}(f)+3 \cdot \frac{1}{3}=d(f)+1=4$. For any four-face or five-face $f$ of $G, c^{\prime}(f)=\operatorname{ch}(f)=d(f) \geq 4$. For any $6^{+}$-face $f$ of $G$, since $f$ is incident to at most $d(f)$ edges, each of which is incident to a three-face, then $c h^{\prime}(f) \geq \operatorname{ch}(f)-\frac{1}{3} d(f)=\frac{2}{3} d(f) \geq 4$. Therefore, we have:

$$
\sum_{f \in F(G)} c h^{\prime}(f) \geq \sum_{f \in F(G)} 4=4|F(G)|
$$

By Formulae (1) and (2), we have $|E(G)| \geq 2|F(G)|$.

Proof of Theorem 2. Suppose that the theorem is not true, and let $G$ be a counterexample to the theorem. Then, $G$ has at least one three-face and no adjacent $(3, k)$-faces, where $k \leq 5$. By Lemma 1 , $|E(G)| \geq 2|F(G)|$. Using Euler's Formula $|V(G)|-|E(G)|+|F(G)|=2$, we can obtain:

$$
|E(G)| \leq 2|V(G)|-4
$$

Since $G$ is uniquely three-colorable, then by Theorem 1 , we have $|E(G)| \geq 2|V(G)|-3$. This is a contradiction.

Note that the graph shown in Figure 1 is a uniquely three-colorable plane graph having neither adjacent $(3,3)$-faces nor adjacent $(3,4)$-faces. Therefore, the bound of five for $k$ is the best possible.

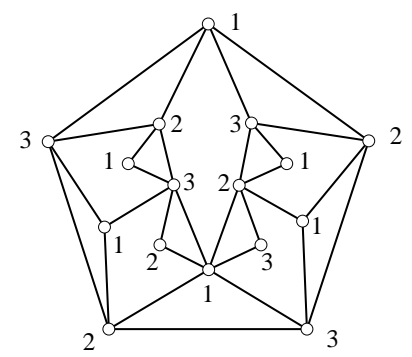

Figure 1. A uniquely three-colorable plane graph having neither adjacent $(3,3)$-faces nor adjacent $(3,4)$-faces.

Remark 1. By piecing together more copies of the plane graph in Figure 1, one can construct an infinite class of uniquely three-colorable plane graphs having neither adjacent $(3,3)$-faces nor adjacent $(3,4)$-faces.

\section{Construction of Uniquely Three-Colorable Plane Graphs without Adjacent $(3,3)$-Faces or Adjacent (3,5)-Faces}

There are many classes of uniquely three-colorable plane graphs having neither adjacent $(3,4)$-faces nor adjacent $(3,5)$-faces, such as even maximal plane graphs (maximal plane graphs in which each vertex has even degree) and maximal outerplanar graphs with at least six vertices. Now, we construct a class of uniquely three-colorable plane graphs having neither adjacent $(3,3)$-faces nor adjacent $(3,5)$-faces and prove that these graphs are edge-critical.

We construct a graph $G_{k}$ as follows: 
(1) $V\left(G_{k}\right)=\left\{u, w, v_{0}, v_{1}, \ldots, v_{3 k-1}\right\}$;

(2) $E\left(G_{k}\right)=\left\{v_{0} v_{1}, v_{1} v_{2}, \ldots, v_{3 k-2} v_{3 k-1}, v_{3 k-1} v_{0}\right\} \cup\left\{u v_{i}: i \equiv 1\right.$ or $\left.2(\bmod 3)\right\} \cup\left\{w v_{i}: i \equiv 0\right.$ or 1 $(\bmod 3)\}$, where $k$ is odd and $k \geq 3$ (see an example $G_{3}$ shown in Figure 2$)$.

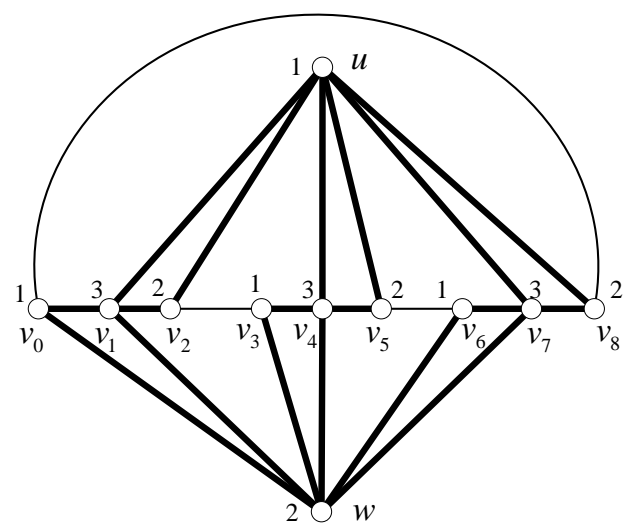

Figure 2. An example $G_{3}$.

Theorem 3. For any odd $k$ with $k \geq 3, G_{k}$ is uniquely three-colorable.

Proof. Let $f$ be any three-coloring of $G_{k}$. Since $v_{0} v_{1} \ldots v_{3 k-1} v_{0}$ is a cycle of odd length and each $v_{i}$ is adjacent to $u$ or $w$, we have $f(u) \neq f(w)$. Without loss of generality, let $f(u)=1$ and $f(w)=2$. By the construction of $G_{k}$, we know that $v_{3 j+1}$ is adjacent to both $u$ and $w$, where $j=0,1, \ldots, k-1$. Therefore, $v_{3 j+1}$ can only receive the color three, namely $f\left(v_{3 j+1}\right)=3, j=0,1, \ldots, k-1$. Since $v_{3 j}$ is adjacent to both $w$ and $v_{3 j}$ in $G_{k}$, we have $f\left(v_{3 j}\right)=1, j=0,1, \ldots, k-1$. Similarly, we can obtain $f\left(v_{3 j+2}\right)=2$, $j=0,1, \ldots, k-1$. Therefore, the three-coloring $f$ is uniquely decided as shown in Figure 2 , and then, $G_{k}$ is uniquely three-colorable.

Theorem 4. For any odd $k$ with $k \geq 3, G_{k}$ is edge-critical.

Proof. To complete the proof, it suffices to show that $G_{k}-e$ is not uniquely three-colorable for any edge $e \in E\left(G_{k}\right)$. Let $f$ be a uniquely three-coloring of $G_{k}$ shown in Figure 2. Denote by $E_{i j}$ the set of edges in $G_{k}$ whose ends are colored by $i$ and $j$, respectively, where $1 \leq i<j \leq 3$. Namely:

$$
E_{i j}=\left\{x y: x y \in E\left(G_{k}\right), f(x)=i, f(y)=j\right\}, 1 \leq i<j \leq 3 .
$$

Observation 1 . Both the subgraphs $G_{k}\left[E_{13}\right]$ and $G_{k}\left[E_{23}\right]$ of $G_{k}$ induced by $E_{13}$ and $E_{23}$ are trees. Observation 2. The subgraph $G_{k}\left[E_{12}\right]$ of $G_{k}$ induced by $E_{12}$ consists of $k$ internally disjoint paths $u v_{3 i-1} v_{3 i} w$, where $i=1,2, \ldots, k$.

If $e \in E_{13} \cup E_{23}$, then $G_{k}-e$ is not uniquely three-colorable by Observation 1. Suppose that $e \in E_{12}$. By Observation 2, there exists a number $t \in\{1,2, \ldots, k\}$ such that $e \in\left\{u v_{3 t-1}, v_{3 t-1} v_{3 t}, v_{3 t} w\right\}$. Moreover, $G_{k}-e$ contains at least one vertex of degree two. By repeatedly deleting vertices of degree two in $G_{k}-e$, we can obtain a subgraph $G_{k}-\left\{v_{3 t-1}, v_{3 t}\right\}$ of $G_{k}$. Now, we prove that $G_{k}-\left\{v_{3 t-1}, v_{3 t}\right\}$ is not uniquely three-colorable.

It can be seen that the restriction $f_{0}$ of $f$ to the vertices of $G_{k}-\left\{v_{3 t-1}, v_{3 t}\right\}$ is a three-coloring of $G_{k}-\left\{v_{3 t-1}, v_{3 t}\right\}$. On the other hand, $G_{k}-\left\{v_{3 t-1}, v_{3 t}, u, w\right\}$ is a path, denoted by $P$. Let $f^{\prime}(u)=f^{\prime}(w)=1$, and alternately, color the vertices of $P$ by the other two colors. We can obtain a three-coloring $f^{\prime}$ of $G_{k}-\left\{v_{3 t-1}, v_{3 t}\right\}$, which is distinct from $f_{0}$. Since each three-coloring of $G_{k}-\left\{v_{3 t-1}, v_{3 t}\right\}$ can be extended to a three-coloring of $G_{k}-e$, we know that $G_{k}-e$ is not uniquely three-colorable when $e \in E_{12}$.

Since $E\left(G_{k}\right)=E_{12} \cup E_{13} \cup E_{23}, G_{k}-e$ is not uniquely three-colorable for any edge $e \in E\left(G_{k}\right)$. 
Note that $G_{k}$ has $3 k+2$ vertices and $7 k$ edges by the construction. From Theorem 4 , we can obtain the following result.

Corollary 1. There exists an infinite family of edge-critical uniquely three-colorable plane graphs with $n$ vertices and $\frac{7}{3} n-\frac{14}{3}$ edges, where $n(\geq 11)$ is odd and $n \equiv 2(\bmod 3)$.

Denote by size(n) the upper bound of the number of edges of edge-critical uniquely three-colorable planar graphs with $n$ vertices. Then, by Corollary 1 and the result due to Li et al. [13], we can obtain the following result.

Corollary 2. For any odd integer $n$ such that $n \equiv 2(\bmod 3)$ and $n \geq 11$, we have $\frac{7}{3} n-\frac{14}{3} \leq \operatorname{size}(n) \leq \frac{5}{2} n-6$.

Proof. First, in [13], Li et al. proved that $\operatorname{size}(n) \leq \frac{5}{2} n-6$ for any edge-critical uniquely three-colorable planar graph $G$ with $n(n \geq 6)$ vertices. Then, by Corollary 1 , we can conclude that Corollary 2 is true.

Corollary 2 improves the lower bound $\frac{9}{4} n-6$ of $\operatorname{size}(n)$ given by Matsumoto [17] and gives a negative answer to a problem proposed by Mel'nikov and Steinberg [16], who asked that $\operatorname{size}(n)=\frac{9}{4} n-6$ for any $n \geq 12$.

\section{Conclusions and Conjectures}

In this paper, we obtained a structural property of uniquely three-colorable plane graphs. We proved that every uniquely three-colorable plane graph has adjacent $(3, k)$-faces, where $k \leq 5$, and the bound of five for $k$ is the best possible. The graph in Figure 1 shows a uniquely three-colorable plane graph having neither adjacent $(3,3)$-faces nor adjacent $(3,4)$-faces. However this plane graph is two-connected. This prompts us to propose the following conjecture.

Conjecture 1. Let $G$ be a three-connected uniquely three-colorable plane graph. Then, $G$ has adjacent $(3, k)$-faces, where $k \leq 4$.

It can be seen that the uniquely three-colorable plane graph $G_{k}$ constructed in Section 3 is three-connected. So Therefore, Conjecture 1 is true, then the bound of four for $k$ is the best possible. Moreover, because the family of graphs $G_{k}$ is the edge-critical uniquely three-colorable planar graphs with the largest number of edges found at present, we recall the follow conjecture proposed by Li et al [13].

Conjecture 2 ([13]). Let $G$ be an edge-critical uniquely three-colorable planar graph with $n$ vertices. Then, $\operatorname{size}(n) \leq \frac{7}{3} n-\frac{14}{3}$.

Author Contributions: Formal analysis, Z.L., N.M., and E.Z.; investigation, Z.L.; methodology, Z.L., N.M., J.X., and T.J.; writing, original draft, Z.L.

Funding: This research was supported by the National Natural Science Foundation of China under Grant Number 61802158 and the Fundamental Research Funds for the Central Universities under Grant Number lzujbky-2018-37.

Conflicts of Interest: The authors declare no conflict of interest. The funders had no role in the design of the study; in the collection, analyses, or interpretation of data; in the writing of the manuscript; nor in the decision to publish the results.

\section{References}

1. Dey, A.; Son, L.H.; Kumar, P.K.K.; Selvachandran, G.; Quek, S.G. New Concepts on Vertex and Edge Coloring of Simple Vague Graphs. Symmetry 2018, 10, 373. [CrossRef] 
2. Orden, D.; Gimenez-Guzman, J.M.; Marsa-Maestre, I.; De la Hoz, E. Spectrum Graph Coloring and Applications to Wi-Fi Channel Assignment. Symmetry 2018, 10, 65. [CrossRef]

3. Yang, L.; Dang, R.; Li, M.; Zhao, K.; Song, C.; Xu, Z. A Fast Calibration Method for Phased Arrays by Using the Graph Coloring Theory. Sensors 2018, 18, 4315. [CrossRef] [PubMed]

4. Bondy, J.A.; Murty, U.S.R. Graph Theory; Springer: Berlin/Heidelberg, Germany, 2008.

5. Birkhoff, G.D. A determinant formula for the number of ways of colouring a map. Ann. Math. 1912, 14, 42-46. [CrossRef]

6. Whitney, H. The coloring of graphs. Ann. Math. 1932, 33, 688-718. [CrossRef]

7. Read, R.C. An introduction to chromatic polynomials. J. Comb. Theory 1968, 4, 52-71. [CrossRef]

8. Harary, F.; Cartwright, D. On the coloring of signed graphs. Elem. Math. 1968, 23, 85-89.

9. Bollobás, B. Uniquely colorable graphs. J. Comb. Theory Ser. B 1978, 25, 54-61. [CrossRef]

10. Chartrand, G.; Geller, D.P. On uniquely colorable planar graphs. J. Comb. Theory 1969, 6, 271-278. [CrossRef]

11. Harary, F.; Hedetniemi, S.T.; Robinson, R.W. Uniquely colorable graphs. J. Comb. Theory 1969, 6, $264-270$. [CrossRef]

12. Li, Z.P.; Zhu, E.Q.; Shao, Z.H.; Xu, J. A note on uniquely 3-colorable planar graphs. Int. J. Comput. Math. 2017, 94, 1028-1035. [CrossRef]

13. Li, Z.P.; Zhu, E.Q.; Shao, Z.H.; Xu, J. Size of edge-critical uniquely 3-colorable planar graphs. Discret. Math. 2016, 339, 1242-1250. [CrossRef]

14. Dailey, D.P. Uniqueness of colorability and colorability of planar 4-regular graphs are NP-complete. Discret. Math. 1980, 30, 289-293. [CrossRef]

15. Aksionov, V.A. On uniquely 3-colorable planar graphs. Discret. Math. 1977, 20, 209-216. [CrossRef]

16. Mel'nikov, L.S.; Steinberg, R. One counterexample for two conjectures on three coloring. Discret. Math. 1977, 20, 203-206. [CrossRef]

17. Matsumoto, N. The size of edge-critical uniquely 3-colorable planar graphs. Electron. J. Comb. 2013, 20, 49.

(C) 2019 by the authors. Licensee MDPI, Basel, Switzerland. This article is an open access article distributed under the terms and conditions of the Creative Commons Attribution (CC BY) license (http:/ / creativecommons.org/licenses/by/4.0/). 